\title{
Application of a Large-Parameter Technique for Solving a Singular Case of a Rigid Body
}

\author{
A. I. Ismail $\mathbb{D}^{1,2}$ \\ ${ }^{1}$ Mechanical Engineering Department, College of Engineering and Islamic Architecture, Umm Al-Qura University, Makkah, \\ P.O. Box 5555, Saudi Arabia \\ ${ }^{2}$ Mathematics Department, Faculty of Science, Tanta University, Tanta, P.O. Box 31527, Egypt
}

Correspondence should be addressed to A. I. Ismail; aiismail@uqu.edu.sa

Received 7 August 2020; Revised 6 December 2020; Accepted 22 February 2021; Published 18 March 2021

Academic Editor: Eugen Radu

Copyright (c) 2021 A. I. Ismail. This is an open access article distributed under the Creative Commons Attribution License, which permits unrestricted use, distribution, and reproduction in any medium, provided the original work is properly cited.

In this paper, the motion of a rigid body in a singular case of the natural frequency $(\omega=1 / 3)$ is considered. This case of singularity appears in the previous works due to the existence of the term $\left[\omega^{2}-(1 / 9)\right]$ in the denominator of the obtained solutions. For this reason, we solve the problem from the beginning. We assume that the body rotates about its fixed point in a Newtonian force field and construct the equations of the motion for this case when $\omega=1 / 3$. We use a new procedure for solving this problem from the beginning using a large parameter $\varepsilon$ that depends on a sufficiently small angular velocity component $r_{o}$. Applying this procedure, we derive the periodic solutions of the problem and investigate the geometric interpretation of motion. The obtained analytical solutions graphically are presented using programmed data. Using the fourth-order Runge-Kutta method, we find the numerical solutions for this case aimed at determining the errors between both obtained solutions.

\section{Introduction}

In [1], the problem of the motion of a fast coherent body around a fixed point under the influence of a Newtonian field of attraction at the value $\omega=1 / 3$ of the natural frequency was studied. This anomaly appeared in [2] and is specialized in various bodies classified according to the inertia. Using the Poincare small-parameter method [3], periodic solutions-with basic zero amplitudes-of the semilinear independent system were obtained in the form of power series expansions up to the third approximation containing assumed small parameters. In [4], Poincare's method that depends on a small parameter assumed to be inversely proportional to a high angular velocity component $r_{o}$ is studied. Some important other analytical and numerical solutions besides practical applications are presented in $[5,6]$.

In our problem, we assume a slowly rotating body with low angular velocity $r_{o}$, so we cannot use the smallparameter technique. We must look for another technique to solve the considered problem under the new initial conditions of the motion. We assume a large parameter $\varepsilon$ directly proportional to $1 / r_{o}$ [7]. Therefore, we qualify the large- parameter method [8] to solve the presented problem under the new initial conditions for the motion. The equations of motion and their reduction are derived to describe the behavior of the body at any instant in time. The first integrals of the motion are obtained. Achieving a large parameter depends on the properties of the motion, and the periodic solutions are obtained in a new domain using the large-parameter technique. The geometric interpretation of the motion is illustrated to describe Euler's angles in a new domain depending on the time, the angular velocity, and the large parameter. Gyroscopic motions are considered for some values of moments of inertia. Many applications are given.

\section{The Considered Problem}

The semilinear system of equations of motions is obtained in suitable symbols in [7] as follows:

$$
\begin{aligned}
& 9 \ddot{p}_{2}+p_{2}-9 \varepsilon^{-2} F\left(p_{2}, \dot{p}_{2}, \gamma_{2}, \dot{\gamma}_{2}, \varepsilon\right)=0, \\
& \ddot{\gamma}_{2}+\gamma_{2}-\varepsilon^{-2} \phi\left(p_{2}, \dot{p}_{2}, \gamma_{2}, \dot{\gamma}_{2}, \varepsilon\right)=0,
\end{aligned}
$$


TABLE 1: The analytical solutions when $\omega=1 / 3$.

\begin{tabular}{lcccc}
\hline$t$ & $P$ & $J$ & $X_{1}$ & $Y_{1}$ \\
\hline 0 & 1.154701 & 3.179794 & $6.67 E-01$ & $2.13 E-11$ \\
10 & 1.544112 & 2.576352 & $5.72 E-01$ & -1.86373 \\
20 & 1.866479 & $9.95 E-01$ & $4.53 E-01$ & -3.02009 \\
30 & 2.107803 & $-9.64 E-01$ & $3.15 E-01$ & -3.03018 \\
40 & 2.257608 & -2.55702 & $1.62 E-01$ & -1.89017 \\
50 & 2.309387 & -3.17963 & $2.64 E-03$ & $-3.28 E-02$ \\
60 & 2.260894 & -2.59541 & $-1.57 E-01$ & 1.837096 \\
70 & 2.114234 & -1.02612 & $-3.10 E-01$ & 3.009679 \\
80 & 1.875774 & $9.33 E-01$ & $-4.49 E-01$ & 3.039946 \\
90 & 1.555868 & 2.537413 & $-5.69 E-01$ & 1.916409 \\
100 & 1.168407 & 3.179119 & $-6.64 E-01$ & $6.55 E-02$ \\
110 & $7.30 E-01$ & 2.614198 & $-7.30 E-01$ & -1.81027 \\
120 & $2.60 E-01$ & 1.057064 & $-7.65 E-01$ & -2.99895 \\
130 & $-2.21 E-01$ & $-9.01 E-01$ & $-7.66 E-01$ & -3.04939 \\
140 & $-6.92 \mathrm{E}-01$ & -2.51754 & $-7.34 E-01$ & -1.94245 \\
150 & -1.13404 & -3.17828 & $-6.71 E-01$ & $-9.82 E-02$ \\
160 & -1.52634 & -2.63271 & $-5.78 E-01$ & 1.783242 \\
170 & -1.85237 & -1.0879 & $-4.60 E-01$ & 2.987903 \\
180 & -2.09797 & $8.70 E-01$ & $-3.22 E-01$ & 3.058513 \\
190 & -2.25248 & 2.497397 & $-1.70 E-01$ & 1.968273 \\
200 & -2.30918 & 3.177095 & $-1.06 E-02$ & $1.31 E-01$ \\
210 & -2.26562 & 2.650935 & $1.49 E-01$ & -1.75603 \\
220 & -2.12369 & 1.118617 & $3.02 E-01$ & -2.97654 \\
230 & -1.88955 & $-8.38 E-01$ & $4.43 E-01$ & -3.06731 \\
240 & -1.57337 & -2.47699 & $5.64 E-01$ & -1.99389 \\
250 & -1.18886 & -3.17558 & $6.60 E-01$ & $-1.64 E-01$ \\
260 & $-7.53 E-01$ & -2.66888 & $7.28 E-01$ & 1.72863 \\
270 & $-2.84 E-01$ & -1.14922 & $7.64 E-01$ & 2.964859 \\
280 & $1.97 E-01$ & $8.07 E-01$ & $7.67 E-01$ & 3.075783 \\
290 & $6.70 E-01$ & 2.45632 & $7.37 E-01$ & 2.019302 \\
300 & 1.113256 & 3.173723 & $6.74 E-01$ & $1.96 E-01$ \\
\hline & & & &
\end{tabular}

where

$$
\begin{aligned}
p_{2} & =p_{2}(p, \gamma), \quad\left(p_{2} \gamma_{2}\right), \quad=\frac{d}{d \tau}, \quad \tau=\frac{t}{r_{o}}, \\
F & =F_{2}+\varepsilon^{-1} F_{3}+\cdots,(F \phi), \varepsilon=c \frac{\sqrt{\gamma_{o}{ }^{\prime \prime}}}{r_{0}}, \quad 0<\gamma_{o}^{\prime \prime} \prec 1, \\
F_{k}(\tau) & =F_{k}\left(p_{2}^{(0)}, \dot{p}_{2}^{(0)}, \gamma_{2}^{(0)}, \dot{\gamma}_{2}^{(0)}\right) \equiv F_{k}^{(0)},(F \phi),(k=2,3) .
\end{aligned}
$$

The symbols like $(a b)$ denote the canceled equations; $c$ is a constant.

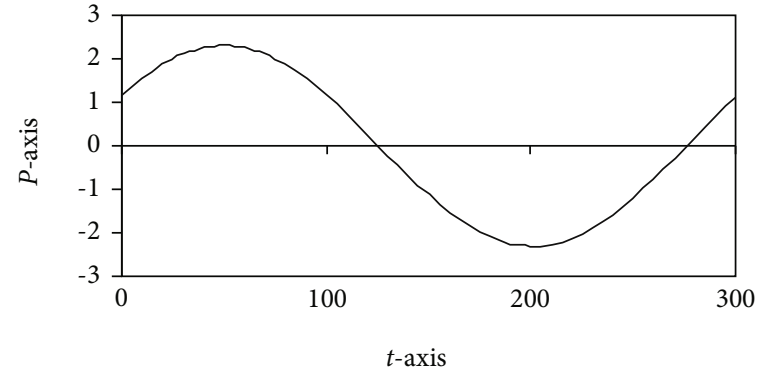

Figure 1: The analytical solution $P$ against the time $t$.

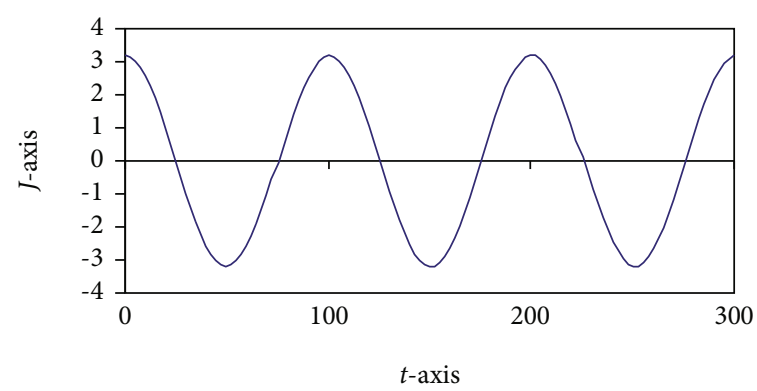

FIgURE 2: The analytical solution $J$ against the time $t$.

The following first integral is deduced as [9]

$$
\begin{aligned}
\gamma_{2}^{2}= & 1-\gamma_{0}^{\prime \prime-2}+\dot{\gamma}_{2}^{2}+2 \varepsilon^{-1}\left(v p_{2} \gamma_{2}+v_{1} \dot{p}_{2} \dot{\gamma}_{2}+s_{21}\right)+\varepsilon^{-2} \\
& \cdot\left[v_{1}^{2} \dot{p}_{2}^{2}-2 \dot{\gamma}_{2} A_{1}^{-1}\left(e_{1} \dot{\gamma}_{2}+\dot{p}_{2} s_{21}+\frac{0.5 \dot{\gamma}_{2} s_{11}}{A_{1}}-y_{0}^{\prime} a^{-1}\right)\right. \\
& \left.+v^{2} p_{2}^{2}+s_{21}^{2}+\left(2 s_{22}-s_{11}\right)\right]+\varepsilon^{-3}(\cdots),
\end{aligned}
$$

where

$$
S_{i j}^{(0)}=S_{i j}^{(0)}\left(p_{2}^{(0)}, \dot{p}_{2}^{(0)}, \gamma_{2}^{(0)}, \dot{\gamma}_{2}^{(0)}\right) \quad(i, j=1,2),
$$

$v, v_{1}, A_{1}, e_{1}, y_{o}{ }^{\prime}$, and $a$ are constants depending on the rigid body parameters.

Applying the method of the large parameter [8], we obtain periodic solutions-with basic zero amplitudes-of the semilinear independent system in the form of power series expansions until the second approximation. On the use of a hypothetical large parameter, we achieve the periodic solutions in the following form:

$$
\begin{aligned}
p= & c \sqrt{\gamma_{0}{ }^{\prime \prime}}\left\{\left(M_{1} \cos 0.33 \tau+M_{2} \sin 0.33 \tau\right)\right. \\
& +\varepsilon^{-1}\left(\chi+\ell_{1} \cos 0.33 \tau+m_{1} \sin 0.33 \tau+\chi_{1} M_{3} \cos \tau\right) \\
& \left.+\varepsilon^{-2} \sum_{i=0}^{7}\left(R_{1 i} \cos 0.33 i \tau+R_{1 i}^{\prime} \sin 0.33 i \tau\right)+\cdots\right\}, \quad i \neq 6,
\end{aligned}
$$


TABLE 2: The numerical solutions when $\omega=1 / 3$.

\begin{tabular}{|c|c|c|c|c|}
\hline$t$ & $P$ & $J$ & $X_{1}$ & $Y_{1}$ \\
\hline 0 & 1.154701 & 3.179794 & $6.67 E-01$ & $2.13 E-11$ \\
\hline 10 & 1.544319 & 2.576409 & $5.73 E-01$ & -1.863584 \\
\hline 20 & 1.866884 & $9.95 E-01$ & $4.54 E-01$ & -3.019916 \\
\hline 30 & 2.108389 & $-9.63 E-01$ & $3.15 E-01$ & -3.030202 \\
\hline 40 & 2.258349 & -2.556521 & $1.62 E-01$ & -1.890568 \\
\hline 50 & 2.310252 & -3.179412 & $2.81 E-03$ & $-3.35 E-02$ \\
\hline 60 & 2.261844 & -2.595745 & $-1.57 E-01$ & 1.8362 \\
\hline 70 & 2.115229 & -1.027045 & $-3.10 E-01$ & 3.00906 \\
\hline 80 & 1.876771 & $9.31 E-01$ & $-4.49 E-01$ & 3.039994 \\
\hline 90 & 1.556824 & 2.536283 & $-5.69 E-01$ & 1.917292 \\
\hline 100 & 1.169281 & 3.178677 & $-6.64 E-01$ & $6.70 E-02$ \\
\hline 110 & $7.31 E-01$ & 2.61479 & $-7.31 E-01$ & -1.808615 \\
\hline 120 & $2.61 E-01$ & 1.058642 & $-7.65 E-01$ & -2.997872 \\
\hline 130 & $-2.20 E-01$ & $-8.99 E-01$ & $-7.67 E-01$ & -3.049446 \\
\hline 140 & $-6.92 E-01$ & -2.515767 & $-7.35 E-01$ & -1.943798 \\
\hline 150 & -1.13402 & -3.177588 & $-6.71 E-01$ & $-1.01 E-01$ \\
\hline 160 & -1.52653 & -2.633542 & $-5.78 E-01$ & 1.780833 \\
\hline 170 & -1.85276 & -1.090117 & $-4.60 E-01$ & 2.986351 \\
\hline 180 & -2.09855 & $8.67 E-01$ & $-3.22 E-01$ & 3.058559 \\
\hline 190 & -2.25321 & 2.494973 & $-1.70 E-01$ & 1.970085 \\
\hline 200 & -2.31004 & 3.176146 & $-1.07 E-02$ & $1.34 E-01$ \\
\hline 210 & -2.26657 & 2.651998 & $1.49 E-01$ & -1.752857 \\
\hline 220 & -2.12469 & 1.121468 & $3.02 E-01$ & -2.9745 \\
\hline 230 & -1.89055 & $-8.35 E-01$ & $4.43 E-01$ & -3.06733 \\
\hline 240 & -1.57433 & -2.473905 & $5.64 E-01$ & -1.996149 \\
\hline 250 & -1.18975 & -3.174351 & $6.60 E-01$ & $-1.67 E-01$ \\
\hline 260 & $-7.54 E-01$ & -2.670157 & $7.28 E-01$ & 1.724689 \\
\hline 270 & $-2.85 E-01$ & -1.152689 & $7.64 E-01$ & 2.96232 \\
\hline 280 & $1.97 E-01$ & $8.02 E-01$ & $7.67 E-01$ & 3.075759 \\
\hline 290 & $6.70 E-01$ & 2.452564 & $7.37 E-01$ & 2.021988 \\
\hline 300 & 1.113222 & 3.172203 & $6.75 E-01$ & $2.01 E-01$ \\
\hline
\end{tabular}

$$
\begin{aligned}
q= & c \sqrt{\gamma_{0}^{\prime \prime}}\left\{0.33 A_{1}^{-1}\left(M_{1} \sin 0.33 \tau-M_{2} \cos 0.33 \tau\right)\right. \\
& +A_{1}^{-1} \varepsilon^{-1}\left[y_{0}^{\prime} a^{-1}+\chi_{2} M_{3} \sin \tau-0.33\left(\ell_{1} \sin 0.33 \tau+m_{1} \cos 0.33 \tau\right)\right] \\
& \left.+\varepsilon^{-2} \sum_{i=0}^{7}\left(R_{2 i} \cos 0.33 i \tau+R_{2 i}^{\prime} \sin 0.33 i \tau\right)+\cdots\right\}, \quad i \neq 6, \\
r= & r_{0}\left\{1+0.5 E^{2} \varepsilon^{-2}\left[\sum_{i=0}^{7}\left(R_{3 i} \cos 0.33 i \tau+R_{3 i}^{\prime} \sin 0.33 i \tau\right)+R_{36} \cos 2 \tau\right]+\cdots\right\}, \quad i \neq 1, \\
\gamma= & \gamma_{0}^{\prime \prime}\left\{M_{3} \cos \tau+\varepsilon^{-1} a\left[M_{1} \cos 0.33 \tau+M_{2} \sin 0.33 \tau\right)-M_{1} \cos \tau\right] \\
& \left.+\varepsilon^{-2}\left[\sum_{i=0}^{6}\left(R_{4 i} \cos 0.33 \tau+R_{4 i}^{\prime} \sin 0.33 \tau\right)+R_{49} \cos 3 \tau\right]+\cdots\right\}, \quad i \neq 2,4 \\
\gamma^{\prime}= & \gamma_{0}^{\prime \prime}\left\{-M_{3} \sin \tau-\varepsilon^{-1} a\left[0.5 v_{1}\left(M_{1} \sin 0.33 \tau-M_{2} \cos 0.33 \tau\right)-a M_{1} \sin \tau\right]\right. \\
& \left.+\varepsilon^{-2}\left[\sum_{i=0}^{6}\left(R_{5 i} \cos 0.33 i \tau+R_{5 i}^{\prime} \sin 0.33 i \tau\right)+R_{59} \sin 3 \tau\right]+\cdots\right\}, \quad i \neq 2,4
\end{aligned}
$$

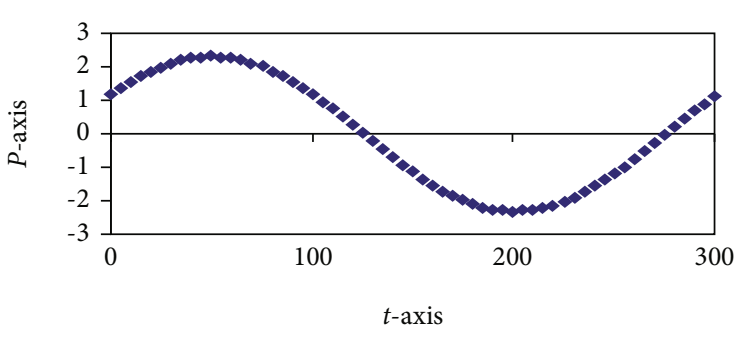

Figure 3: The numerical solution $P$ against the time $t$.

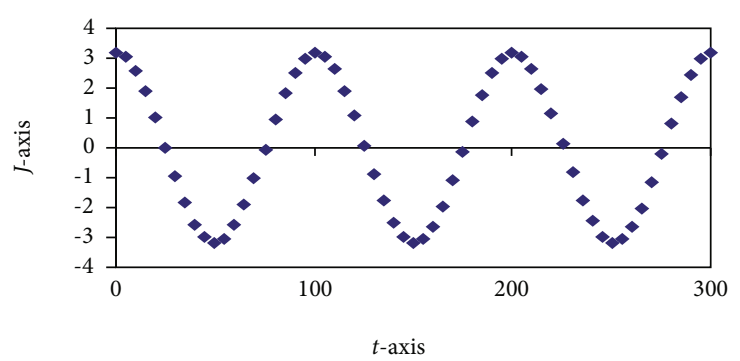

Figure 4: The numerical solution $J$ against the time $t$.

$$
\begin{aligned}
\gamma^{\prime \prime}= & \gamma_{0}^{\prime \prime}\left\{1+\varepsilon^{-1} M_{3} E\left[a \cos \delta+0.5\left(0.33 b A_{1}^{-1}-a\right) \cos (\delta+0.66 \tau)\right.\right. \\
& \left.-0.5\left(0.33 b A_{1}^{-1}+a\right) \cos (\delta-1.25 \tau)\right] \\
& \left.+\varepsilon^{-2}\left[\sum_{i=0}^{4}\left(R_{6 i} \cos 0.33 i \tau+R_{6 i}^{\prime} \sin 0.33 i \tau\right)+R_{66} \cos 2 \tau\right]+\cdots\right\}, \quad i \neq 1,
\end{aligned}
$$

where $M_{1}, M_{2}, M_{3}$ are the basic amplitudes of the initial solutions of $p_{2}, \dot{p}_{2}, \gamma_{2}$, respectively, $\ell_{1}, m_{1}$ are the deviations of the amplitudes of the solutions $p_{2}, \dot{p}_{2}$ from their basic amplitudes, $E=\left(M_{1}^{2}+M_{2}^{2}\right)^{1 / 2}, \delta=\tan ^{-1}\left(M_{2} / M_{1}\right)$, and $c, \chi, \chi_{1}, \chi_{2}$, and $R_{j i}$ are constants that depend on the rigid body parameter and are determined with correspondence by the system (1).

\section{Geometric Interpretation of Motion}

In this section, we describe the motion by Euler's angles $\theta, \psi$, and $\varphi$ which are determined from the obtained periodic solutions (5). Since the initial system of (1) does not depend explicitly on time, then the periodic solutions are still so if $t$ is replaced by $\left(t+t_{0}\right)$ where $t_{0}$ is an arbitrary interval of time [10]. Let $t=t_{0}$ be the initial instant of time, and substituting the periodic solutions (5) into Euler's angles, we get [11]

$$
\begin{aligned}
\varphi_{0}-0.5 \pi= & r_{0} t_{0}+\cdots, \tan \theta_{0}=M_{3}, \\
\theta-\theta_{0}= & \varepsilon^{-1} E\left[\theta_{1}\left(t+t_{0}\right)-\theta_{1}\left(t_{0}\right)\right]-\varepsilon^{-2} \cos \theta_{0} \sin ^{-1} \theta_{0} \\
& \cdot\left[\theta_{2}\left(t+t_{0}\right)-\theta_{2}\left(t_{0}\right)\right]+\cdots, \\
\psi-\psi_{0}= & M g \ell C^{-1} r_{0}{ }^{-1} \sin ^{-1} \theta_{0}\left(0.5 \chi_{1}+v_{0} \cos ^{2} \theta_{0} \sin ^{-1} \theta_{0}\right) t \\
& +0.5 \varepsilon^{-1} r_{0} \sin ^{-1} \theta_{0}\left[\psi_{1}\left(t+t_{0}\right)-\psi_{1}\left(t_{0}\right)\right] \\
& +\varepsilon^{-2} r_{0} \cos \theta_{0} \sin ^{-3} \theta_{0}\left[\psi_{2}\left(t+t_{0}\right)-\psi_{2}\left(t_{0}\right)\right]+\cdots,
\end{aligned}
$$




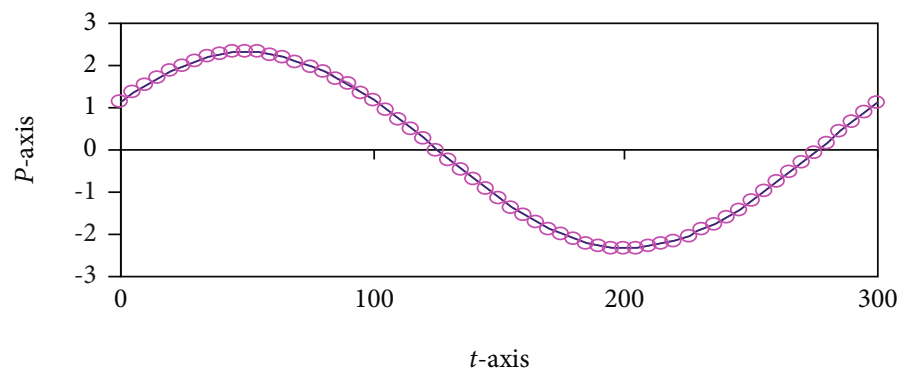

Figure 5: The analytical and numerical solutions $P$ against the time $t$.

$$
\begin{aligned}
\varphi-\varphi_{0}= & \left\{r_{0}-0.5 \chi_{1} M g l C^{-1} r_{0}^{-1}\left(\gamma_{o}{ }^{\prime \prime} \sin ^{-1} \theta_{0}+a E r_{0}{ }^{-1} c \cos \delta \sqrt{\gamma_{o}^{\prime \prime}}\right)\right. \\
& +0.5 M g l C^{-1} E^{2} R_{30} \gamma_{o}^{\prime \prime}-v_{0}^{*} \sqrt{\gamma_{o}{ }^{\prime \prime}}\left[r_{0}+a c E \cos \delta \sin \theta_{0} / \sqrt{\gamma_{o}^{\prime \prime}}\right. \\
& \left.+M g l C^{-1} r_{0}{ }^{-1} R_{60} \gamma_{o}{ }^{\prime \prime}\right]-0.17 E M g l C^{-1} \sqrt{\gamma_{o}^{\prime \prime}}\left[0.5\left(0.33 b A_{1}^{-1}-a\right)\right. \\
& \left.\left.\cdot\left(R_{72}^{\prime} \cos \delta+R_{72} \sin \delta\right)-\left(0.33 b A_{1}^{-1}+a\right)\left(R_{74}^{\prime} \cos \delta-R_{74} \sin \delta\right)\right]\right\} t \\
& +0.5 \varepsilon^{-1} \sqrt{\gamma_{o}^{\prime \prime}}\left[\varphi_{1}\left(t+t_{0}\right)-\varphi_{1}\left(t_{0}\right)\right]+\varepsilon^{-2}\left[\varphi_{2}\left(t+t_{0}\right)-\varphi_{2}\left(t_{0}\right)\right]+\cdots,
\end{aligned}
$$

where the functions $\theta_{1}, \theta_{2}, \theta_{3}, \psi_{1}, \psi_{2}, \varphi_{1}, \varphi_{2}, v_{0}, v_{0}^{*}$ and the constants $R_{i j}, R_{i j}^{\prime}$ are determined from the correspondence with the system (1) and (5).

\section{Numerical and Analytical Solutions of the Slow Spinning Motion of a Body When $\omega=1 / 3$}

In this section, we will program the analytical solutions to the problem mentioned above and represent these solutions graphically. Also, the numerical solutions of the system of semilinear differential equations are considered and will be inferred by the Runge-Kutta method [12] of the fourth rank. The obtained results are represented graphically under the same conditions. In the end, we have done comparing analytical solutions with numerical ones through common graphical representations. We will denote $p_{2}, \gamma_{2}$ by $P, J$, respectively, and their derivatives to time by the symbols $X_{1}=d P / d t, Y_{1}=d J / d t$.

4.1. The Analytical Solutions. Let us introduce the variables [13]:

$$
p=c \sqrt{\gamma_{0}^{\prime \prime}} p_{1}, \gamma=\gamma_{0}^{\prime \prime} \gamma_{1}
$$

then, using (7) and (5), we get

$$
\begin{aligned}
p_{1}= & M_{1} \cos 0.33 \tau+M_{2} \sin 0.33 \tau+\varepsilon^{-1} \\
& \cdot\left(\chi+\ell_{1} \cos 0.33 \tau+m_{1} \sin 0.33 \tau+\chi_{1} M_{3} \cos \tau\right) \\
& +\varepsilon^{-2} \sum_{i=0}^{7}\left(R_{1 i} \cos 0.33 i \tau+R_{1 i}^{\prime} \sin 0.33 i \tau\right)+\cdots,
\end{aligned}
$$

$$
\begin{aligned}
\gamma_{1}= & M_{3} \cos \tau+\varepsilon^{-1} a\left(M_{1} \cos 0.33 \tau+M_{2} \sin 0.33 \tau-M_{1} \cos \tau\right) \\
& +\varepsilon^{-2}\left[\sum_{i=0}^{6}\left(R_{4 i} \cos 0.33 i \tau+R_{4 i}^{\prime} \sin 0.33 i \tau\right)+R_{49} \cos 3 \tau\right]+\cdots,
\end{aligned}
$$

thus, the analytical solutions $p_{2}, \gamma_{2}$ are achieved using a computer program (see Table 1). These solutions appear in the form of numerical values dependent on time and the parameters of the coherent body. Assume the following data of the parameters of the slow spinning gyro obtained for this problem:

$$
\begin{aligned}
A & =2.4, \\
B & =2.4, \\
C & =3.2, \\
x_{0} & =0.0, \\
y_{0} & =0.0, \\
z_{0} & =3.0, \\
\gamma_{0}^{\prime \prime} & =0.3, \\
\lambda & =0.5, \\
r_{0} & =0.000005, \\
T & =18787751.42 E-6 .
\end{aligned}
$$

We obtain the following graphical representations of the analytical solutions (see Figures 1 and 2).

4.2. The Numerical Solutions. Using the semilinear system (1), using the same data (9) and the initial values for the analytical solutions, and then applying the fourth-order Runge-Kutta method through another program, we get the numerical solutions as in Table 2, while the representations of their graphs are shown (see Figures 3 and 4).

To verify the accuracy of the analytical solutions and numerical ones, we compare them graphically (see Figures 5 and 6).

This comparison showed that the deviations between analytical and numerical solutions are very small and can be neglected, and this confirms the accuracy of analytical large-parameter technique and numerical one. 


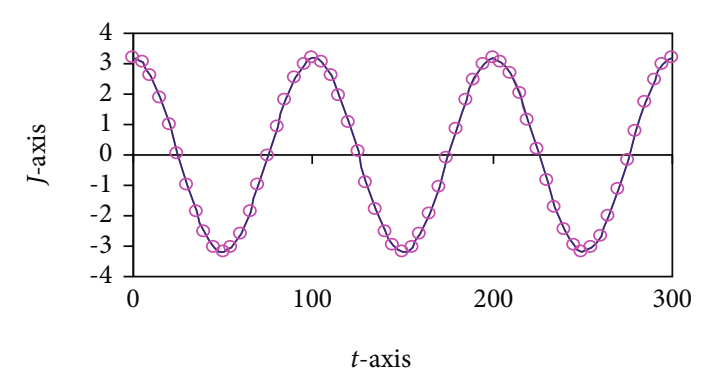

FIGURE 6: The analytical and numerical solutions $J$ against the time $t$.

From the figures, we deduce that the periodicity of the solution $J$ is faster than that for the solution $P$. Moreover, the amplitude for solution $J$ is larger than that for the solution $P$.

\section{Result and Discussion}

The analytical and numerical solutions, their derivatives, and the comparisons between them are given in Tables 1 and 2 and Figures 1-6 which lead us to the following analysis and discussion. Solution $P$ rotates slowly, but solution $J$ rotates fastly. The amplitude of $P$ is between $(-3,3)$ while the amplitude of $J$ is between $(-4,4)$; that is, the amplitude of $J$ is larger than that for $P$.

\section{Conclusions}

We conclude that the equations of motion for a singular case excluded from the previous works [14] are obtained and reduced to a semilinear system of the second order of two variables. New initial conditions of the motion are assumed like the sufficiently small angular velocity of the body given initially about the major of the minor axis of the ellipsoid of inertia. Due to this assumption, we obtain a new parameter named the large parameter. A new procedure named the large-parameter technique is given for solving the obtained system. The geometric interpretations of motions are illustrated in this case. Using the fourth-order Runge-Kutta method, we obtained the numerical solutions for the problem. A comparison between the analytical and the numerical solutions is carried out to prove the accuracy of both techniques and solutions obtained. The errors between both techniques are very small and can be neglected. It is possible to enter the third component of the gyroscopic moment vector on the movement of the body and deduce the effect of this rotation on the body as well as its engineering interpretation. All anomalies that appeared in previous researches $[15,16]$ have been treated. The Lagrange gyroscope as a very special case could also be deduced from the solutions obtained [17]. The considered techniques give the solutions in a new domain of motion which is the reflection of one of the previous works. The numerical and analytical procedures which are presented in this article can be applied for solving other problems by reflecting the parameters of the initial condition of the motion [18-20]. The critical points for this work are summarized as follows:

(1) The natural frequency value $\omega=1 / 3$ treats a permanent singular case in the previous works which cannot be removed

(2) The angular velocity component $r_{o}$ about the $z$-axis is assumed to be sufficiently small instead of sufficiently large value in the previous works

(3) The solutions $p, q, r ; \gamma, \gamma^{\prime}, \gamma^{\prime \prime}$, and Euler's angles $\theta, \psi, \varphi$ are determined in a new domain $\left(\tau \longrightarrow \infty, r_{o} \longrightarrow 0, \varepsilon\right.$ $\longrightarrow \infty)$ instead of $\left(\tau \longrightarrow 0, r_{o} \rightarrow \infty, \varepsilon \longrightarrow 0\right)$ in the previous works

(4) The required initial energy for the motion is low compared with that in the previous works, and the spin is weak which gives a weak oscillation case

(5) The parameter $\varepsilon$ is large instead of a small one in the previous works

(6) The body mass center is slightly displaced from the origin

\section{Nomenclature}

OXYZ: $\quad$ The fixed frame in space

Oxyz: $\quad$ The moving frame fixed in the body

$\omega: \quad$ The natural frequency value

$r_{o}$ : The angular velocity component about the $z$ -axis

$p, q, r: \quad$ The components of the angular velocity vector about the principal axes

$\gamma, \gamma^{\prime}, \gamma^{\prime \prime}$ : The components of the fixed unit vector in the direction of the $Z$-axis

$t: \quad$ The time of the motion

$\theta, \psi, \varphi: \quad$ Euler's angles

$\varepsilon: \quad$ The large parameter

$A, B, C$ : The inertia moments

$\left(x_{o}, y_{o}, z_{o}\right)$ : The body mass center coordinates

$\lambda: \quad$ The Newtonian attracting center coefficient

T: $\quad$ The periodic time

M: $\quad$ The mass of the body

$g$ : The acceleration of the gravity

$\ell: \quad$ The distance between the mass center and the fixed point $O$.

\section{Data Availability}

Data sharing is not applicable to this article as no datasets were generated or analyzed during the current study.

\section{Conflicts of Interest}

The author declares that he has no conflicts of interest. 


\section{References}

[1] A. I. Ismail, "The motion of fast spinning rigid body about a fixed point with definite natural frequency," Aerospace Science and Technology, vol. 1, no. 3, pp. 183-190, 1997.

[2] T. S. Amer, A. I. Ismail, and W. S. Amer, "Application of the Krylov-Bogoliubov-Mitropolski technique for a rotating heavy solid under the influence of a gyrostatic moment," Journal of Aerospace Engineering, vol. 25, no. 3, pp. 421-430, 2012.

[3] T. S. Amer and I. M. Abady, "On the motion of a gyro in the presence of a Newtonian force field and applied moments," Mathematics and Mechanics of Solids, vol. 23, no. 9, pp. 1263-1273, 2017.

[4] T. S. Amer and I. M. Abady, "Solutions of Euler's dynamic equations for the motion of a rigid body," Journal of Aerospace Engineering, vol. 30, no. 4, article 04017021, 2017.

[5] M. Sheikholeslami, M. Nimafar, and D. D. Ganji, "Nanofluid heat transfer between two pipes considering Brownian motion using AGM," Alexandria Engineering Journal, vol. 56, no. 2, pp. 277-283, 2017.

[6] M. M. Peiravi, J. Alinejad, D. Ganji, and S. Maddah, "Numerical study of fins arrangement and nanofluids effects on threedimensional natural convection in the cubical enclosure," Transport Phenomena in Nano and Micro Scales, vol. 7, no. 2, pp. 97-112, 2019.

[7] A. I. Ismail, "Solving a problem of rotary motion for a heavy solid using the large parameter method," Advances in Astronomy, vol. 2020, Article ID 2764867, 7 pages, 2020.

[8] A. H. Nayfeh, Introduction to perturbation techniques, WILEY-VCH Verlag GmbH \& Co. KGaA, 2011.

[9] T. S. Amer and W. S. Amer, "Substantial condition for the fourth first integral of the rigid body problem," Mathematics and Mechanics of Solids, vol. 23, no. 8, pp. 1237-1246, 2018.

[10] Z. A. Sartabanov and B. Z. Omarova, On multi-periodic solutions of quasilinear autonomous systems with an operator of differentiation on the Lyapunov's vector field, K. Zhubanov Aktobe Regional State University, 2019.

[11] S. V. Ershkov and D. Leshchenko, "On a new type of solving procedure for Euler-Poisson equations (rigid body rotation over the fixed point)," Acta Mechanica, vol. 230, no. 3, pp. 871-883, 2019.

[12] V. Ruas, Numerical Methods for Partial Differential Equations: An Introduction, Jhon Wiley \& Sons Ltd, 2016.

[13] T. S. Amer and I. M. Abady, "On the application of the KBM method for the 3-D motion of asymmetric rigid body," Nonlinear Dynamics, vol. 89, no. 3, pp. 1591-1609, 2017.

[14] H. M. Yehia, "On the regular precession of an asymmetric rigid body acted upon by uniform gravity and magnetic fields," Egyptian Journal of Basic and Applied Sciences, vol. 2, no. 3, pp. 200-205, 2015.

[15] F. L. Chernousko, L. D. Akulenko, and D. D. Leshchenko, Evolution of Motions of a Rigid Body About Its Center of Mass, Springer International Publishing AG, 1-12, 2017.

[16] A. V. Borisov and I. S. Mamaev, Rigid Body Dynamics, Higher Education Press, and Walter de Gruyter GmbH, De Gruyter, 2018.

[17] W. S. Amer, "The dynamical motion of a gyroscope subjected to applied moments," Results in Physics, vol. 12, pp. 1429$1435,2019$.
[18] L. Zhang, M. B. Arain, M. M. Bhatti, A. Zeeshan, and H. HalSulami, "Effects of magnetic Reynolds number on swimming of gyrotactic microorganisms between rotating circular plates filled with nanofluids," Applied Mathematics and Mechanics, vol. 41, no. 4, pp. 637-654, 2020.

[19] A. Zeeshan, N. Shehzad, T. Abbas, and R. Ellahi, "Effects of radiative electro-magnetohydrodynamics diminishing internal energy of pressure-driven flow of titanium dioxide-water nanofluid due to entropy generation," Entropy, vol. 21, no. 3, p. 236, 2019.

[20] N. Ijaz, A. Riaz, A. Zeeshan, R. Ellahi, B. Driven, and S. M. Sait, "Buoyancy driven flow with gas-liquid coatings of peristaltic bubbly flow in elastic walls," Journals Coatings, vol. 10, no. 2, p. $115,2020$. 